\title{
Erratum: A New Metric Based Cluster Head Selection Technique for Increasing the Lifetime of Energy Aware Wireless Sensor Network
}

\author{
Sanghita Bhattacharya ${ }^{1}$, Arpan Sarbadhikari ${ }^{2}$, and Subhansu Bandyapadhyay ${ }^{3}$ \\ ${ }^{1}$ Department of Computer Science \& Engineering, National Institute of Technology, \\ Durgapur-713 209, India \\ sanghita.begmail.com \\ 2 A.K. Choudhury School of Information Technology, University of Calcutta, \\ Kolkata-700 009, India \\ arpan1983@yahoo.co.in \\ ${ }^{3}$ Senior member IEEE, Department of Computer Science \& Engineering, \\ University of Calcutta, Kolkata-700 009, India \\ subhansuacomputer.org
}

DOI 10.1007/978-3-642-19542-6_131

In the original version, the name of the first author was spelled incorrectly by mistake. It should be "Sanghita Bhattacharjee". 